\title{
Increased cardiac sympathetic activity: Cause or compensation in vasovagal syncope?
}

\author{
Robert A. Larson ${ }^{1} \cdot$ Mark W. Chapleau ${ }^{1,2,3}$
}

Received: 15 March 2018 / Accepted: 20 March 2018 / Published online: 3 April 2018

(c) Springer-Verlag GmbH Germany, part of Springer Nature (outside the USA) 2018

Vasovagal syncope is relatively common, often occurring during orthostatic stress [1]. Hypotension and loss of consciousness are attributed to inhibition of sympathetic vasoconstrictor tone and/or parasympathetic-mediated bradycardia [1]. While a variety of factors may lead to vasovagal syncope, an initial compensatory increase in sympathetic activity to the heart may paradoxically activate cardiac sensory nerves traveling in the vagus nerve, thereby causing reflex inhibition of sympathetic activity to blood vessels, vasodilation, parasympathetic activation and bradycardia. Chemical factors, either produced endogenously or injected intravascularly, can also activate this reflex, referred to as the Bezold-Jarisch reflex [2]. Chemical factors used experimentally to trigger the reflex include veratrum alkaloids such as veratridine, capsaicin, nicotine, prostanoids, and the serotonin 5- $\mathrm{HT}_{3}$ receptor agonist phenylbiguanide [2].

In this issue of Clinical Autonomic Research, $\mathrm{He}$ and colleagues [3] report that activation of the Bezold-Jarisch reflex with left atrial injection of veratridine in anesthetized dogs elicits two distinct types of neural activity recorded from electrodes placed in the left stellate ganglion: highamplitude spike discharge activity and low-amplitude burst discharge activity. Interestingly, high-amplitude spike discharge increased markedly in some dogs (Group A), but minimally in others (Group B). Moreover, the decreases in blood pressure and heart rate in response to veratridine injection were blunted in Group A dogs compared to Group $\mathrm{B}$ dogs, suggesting that the increased high-amplitude spike discharge activity helped to compensate for the fall in blood

Mark W. Chapleau

mark-chapleau@uiowa.edu

1 Department of Internal Medicine, The University of Iowa Carver College of Medicine, 200 Hawkins Drive, Iowa City, IA 52242, USA

2 Department of Molecular Physiology and Biophysics, University of Iowa, Iowa City, IA 52242, USA

3 Veterans Affairs Medical Center, Iowa City, IA 52246, USA pressure, i.e., it was protective. Consistent with these blunted blood pressure and heart rate responses, plasma norepinephrine levels $30 \mathrm{~s}$ after veratridine injection were significantly higher in Group A dogs.

These intriguing results suggest that a robust sympathoexcitatory response following activation of the Bezold-Jarisch reflex (as occurred in Group A dogs) is compensatory and may predict a smaller fall in blood pressure. And, conversely, a weak sympathoexcitatory response may predict more severe hypotension, and increased risk of syncope. The authors speculate that the results may challenge the use of beta-blockers for treatment of recurrent vasovagal syncope, as beta-blockade would interrupt the compensatory sympathetic response [3].

Of note, the direct measurement of neural activity within the left stellate ganglion by He and colleagues [3] is certainly a unique approach, particularly in regards to studying the Bezold-Jarisch reflex. Little information is available regarding the effect of the Bezold-Jarisch reflex on cardiac sympathetic nerve activity. Salo and colleagues [4] recorded postganglionic cardiac and lumbar sympathetic activity from surgically exposed efferent nerves using hook electrodes in anesthetized rats. The Bezold-Jarisch reflex was activated by phenylbiguanide injected into the right atrium. Interestingly, while lumbar sympathetic nerve activity and heart rate were always decreased by activation of the reflex, cardiac sympathetic nerve activity showed both inhibitory and excitatory responses, with a net excitatory effect [4]. Thus, despite differences in experimental design and species, the findings by $\mathrm{He}$ and colleagues [3] and Salo and colleagues [4] are consistent in terms of observing increased sympathetic activity recorded from stellate ganglia and cardiac sympathetic nerves, respectively. We wonder if the dual inhibitory/ excitatory responses observed by Salo and colleagues [4] might relate to the low-amplitude burst discharge and highamplitude spike discharge reported by $\mathrm{He}$ and colleagues in this issue of Clinical Autonomic Research [3]. 
That said, one must use caution in interpreting the type of sympathetic activity recorded in stellate ganglia. As the authors note in their paper [3], stellate ganglia contain sympathetic neurons that project to other tissues besides the heart [5], including skin, skeletal muscle, and brown adipose tissue. In addition, subtypes of sympathetic neurons, as defined by differences in neurochemical signatures and spike firing properties, innervate the heart [5]. Identification of electrophysiological and neurochemical properties of isolated stellate ganglion neurons retrogradely labeled from the heart would help address some of these issues.

Several questions come to mind. What are the mechanisms driving the high-amplitude spike discharge activity in the left stellate ganglion of Group A dogs and the associated higher blood pressure? Does the arterial baroreceptor reflex contribute to the increased sympathetic nerve activity? Does increased cardiac output mediate the increase in blood pressure? Is spike discharge also increased in right stellate ganglion, which mediates predominantly chronotropic responses? Is there a functional role of changes in low-amplitude burst activity in stellate ganglion?

While questions remain, Dr. He and co-authors are to be commended for their unique approach to recording neural activity in stellate ganglion and the novel finding of two distinct types of sympathetic responses to activation of the Bezold-Jarisch reflex. The suggestion that increased high-amplitude spike discharge activity in stellate ganglion may protect against vasovagal syncope, and that its absence may confer susceptibility to vasovagal syncope, is intriguing and worthy of further investigation.

\section{Compliance with ethical standards}

Conflict of interest None.

Funding National Institutes of Health (P01 HL14388, F32 HL140880).

\section{References}

1. Medow MS, Stewart JM, Sanyal S, Mumtaz A, Sica D, Frishman WH (2008) Pathophysiology, diagnosis, and treatment of orthostatic hypotension and vasovagal syncope. Cardiol Rev 16:4-20

2. Aviado DM, Aviado DG (2001) The Bezold-Jarisch reflex. A historical perspective of cardiopulmonary reflexes. Ann N Y Acad Sci 940:48-58

3. He W, Wang X, Liu S, Yu X, Lu Z, Ma R, Luo D, Xie J, He B, Jiang H (2018) Sympathetic mechanisms in an animal model of vasovagal syncope. Clin Auton Res 24:1-8

4. Salo LM, Woods RL, Anderson CR, McAllen RM (2007) Nonuniformity in the von Bezold-Jarisch reflex. Am J Physiol Regul Integr Comp Physiol 293:R714-R720

5. Mo N, Wallis DI, Watson A (1994) Properties of putative cardiac and non-cardiac neurones in the rat stellate ganglion. J Auton Nerv Syst 47:7-22 